From these experiments, it is seen that under certain discharge conditions a change in the ion species of sulphur hexafluoride is observed, as would indeed be expected. Although no attempt has been made to carry out a rigorous investigation, we have established the fact that appreciable permanent molecular dissociation accompanies the discharge, as shown by the increase in the $\mathrm{F}^{+}$abundance in runs 2 and 3. This increase in $\mathrm{F}^{+}$is to be expected, as the final products should clearly be sulphur and fluorine, but the completeness and speed of the reactions leading to the formation of fluorine are surprising.

The increase in the abundance of $\mathrm{SF}_{2}+$ is unexpected, as it would be thought more likely that the intermediate changes in the reaction $\mathrm{SF}_{6} \rightarrow \mathrm{S}+6 \mathrm{~F}$ would involve changes in the relative abundances of $\mathrm{SF}_{5}+, \mathrm{SF}_{4}+$ and $\mathrm{SF}_{3}+$.

\section{ROBERT THORBURN}

Department of Electrical Engineering,

University, Liverpool.

Nov. 11

${ }^{1}$ Schumb, Trump and Priest, Indust. Eng. Chem. 41, 1348 (1949).

\section{A Molecular Approach to the Problem of Visccelasticity}

As a first step, the type of material considered is that coming under the generic heads of 'paper' and 'board'. These materials are made of cellulosic fibres which are filtered off as sheets from liquid suspensions and dried to give the final product. The bonds between the fibres are almost entirely made up of hydrogen bridges between cellulosic and hemicellulosic components.

The rheological curve obtained may be represented by Fig. 1. The material has all the usual time-effects of viscoelasticity. Under constant load it creeps, and under constant extension it relaxes; consequently it shows hysteresis, and the stress/strain curve is dependent on the rate of application of load or the rate of stretching.

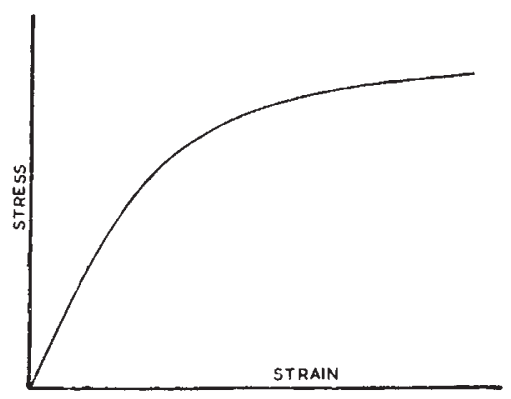

Fig. 1. Stress-strain behaviour of paper

The theory proposed here is that when such a material is strained by $(\delta \sigma)$, showing a stress, $f$, the energy input $(f \delta \sigma)$ simply goes to raise the hydrogen bond potential from $-U_{0}$ at equilibrium to $(U=$ $\left.-U_{0}+\delta U\right)$. Taking infinitesimal quantities, then,

$$
f=\mathrm{d} U / \mathrm{d} \sigma .
$$

It only remains to select a function $U(\sigma)$ to evaluate (1). The Morse ${ }^{1}$ function, rearranged from its usuæl form, is :

$$
U=U_{0}[\exp (-2 a \sigma)-2 \exp (-a \sigma)] .
$$

Thus, differentiating and expanding (2) gives:

$$
\begin{gathered}
f=2 a^{2} U_{0}\left[\sigma-\frac{3}{2} a \sigma^{2}+\frac{7}{6} a^{2} \sigma^{3}---\right] \\
=E \sigma-K \sigma^{2}+----,
\end{gathered}
$$

where $E=2 a^{2} U_{o}$ and $K=3 a^{3} U_{0}$. In these equations $U_{o}$ is in units of energy per unit volume, not per mole.

Equation (3) not only fits the curves obtained for these viscoelastic materials well, but also has the merit of depending on two parameters both of which should be, in principle, deducible from non-rheological data. Thus $a$ can be evaluated rheologically as $(2 K / 3 E)$ or spectroscopically from the infra-red absorption of cellulose. Rheological data give $a=16 \cdot 8 \pm 3$, whereas spectroscopy yields $a=$ $19 \cdot 4 \pm 2$. The agreement may be considered as not unsatisfactory. Physically $a$ is connected with that compressional strain $\sigma^{*}$ which is necessary to raise the hydrogen bridge potential from $-U_{0}$ to zero, by $a=\left(\ln 2 / \sigma^{*}\right)$. Similarly, $U_{o}$ is connected with the molar heat of dissociation of the hydrogen bond $\varphi_{o}$ by $\left[U_{o} / \varphi_{o}\right]=R$, where $R$ is the number of moles of hydrogen bonds per $\mathrm{cm} .^{3}$ of tested material which are subjected to strain.

According to this theory, time-effects are due to the fact that since the hydrogen bond is a weak link it breaks under strain. Thus $R$ continually decreases with time and the problem resolves itself into a problem of chemical kinetics. Re-formation of che weak bonds gives rise to certain appearances of hardening under some conditions.

The choice of the Morse function for the bond potential is somewhat arbitrary; other functions have just as much justification. It is a very convenient function for the purpose; but it suffers from one drawback. This function gives a maximum for $f$ at a strain $\sigma=(\ln 2 / a)$. 'Thus it cannot be applied for materials with $a \sim 20$ beyond a strain of 0.03 or thereabouts. For paper and board this is immaterial, as they normally break at lower strains; for other cellulosic materials this fact sets a limitation on the theory and equations. Cotton yarn, for example, does not yield such useful results as paper and board; probably other functions are required.

I am indebted to Dr. L. Peters, of this Department, for many discussions and for the suggestion to use the Morse function. It is hoped to publish a more detailed treatment of this theory elsewhere.

Department of Textile Industries, Alifred H. Nissan University, Leeds 2. Nov. 8.

1 Morse, P. M., Phys. Rev., 34, 57 (1929).

\section{Infra-red Absorption Spectrum of Fluoro- pyruvic Acid}

Mager and Blank ${ }^{1}$ have reported the synthesis of fluoropyruvic acid and claim that its infra-red spectrum shows an absorption band at $980 \mathrm{~cm} \cdot .^{-1}$, which they assign to the C-F stretching vibration. This frequency is outside the range normally assigned to this vibration ${ }^{2}$, and differs markedly from that suggested $^{3}$ and afterwards found ${ }^{4}$ for this vibration in fluorocitric acid.

An opportunity has recently occurred to examine a sample of fluoropyruvic acid synthesized by the 